\title{
Effect of Coronavirus Disease 2019 Pandemic on Mental Health among Health Care Workers and Others
}

\section{Mona Ahmed Elawady, Marwa Salah El-Dien Abd-Elraouf*}

Public Health Department, Faculty of Medicine, Benha University, Qalyubia, Egypt.

*Corresponding author: Marwa Salah El-Dien Abd-Elraouf, Mobile: (+20) 01065063533, E-mail: marwad24@yahoo.com

\begin{abstract}
Background: A pandemic of Coronavirus 2019 (COVID-19)may endanger the mental well-being of health care workers.

Objective: This is to stipulate some mental issues faced by health care workers at the time of COV-SARS.

Patients and Method: A cross-sectional study was conducted upon 5745 participants from the Qalyubiya Governorate (7 centers). Online self-administered questionnaire (Fear of Coronavirus-19 Scale (7 Qs scale) and The Hospital Anxiety and Depression Scale (HADS) (14 items of two subscales: anxiety and depression)) was utilized. Propensity score matching was performed to minimize bias between health care workers (1740) and non-healthcare workers.

Results: After performing propensity score matching, 1740 participants were included for comparison between health care workers and non-healthcare workers. Health care workers expressed more fear, anxiety, and depression scores. Increasing hours of watching media is a predictor of fear, anxiety, and depression among health care workers $((95 \% \mathrm{CI}$ : $0.555-1.4, \mathrm{p}<.001),(0.77-1.22, \mathrm{p}<.001)$ and $(0.45-0.85, \mathrm{p}<.001)$ respectively) and ((1.44-2.2, $\mathrm{p}<.001),(0.94-1.49$, $\mathrm{p}<.001)$ respectively) among non-healthcare workers.

Conclusions: HCW showed mental disorders scores more than non-healthcare workers. Among health care workers, females, the age group from 26 to 30 years, and more than $4 \mathrm{~h}$ of watching media about COVID-19 had significantly higher mental scores. Non-married health care workers showed a higher fear score. Among non-healthcare workers, married and low educated levels expressed lower scores.
\end{abstract}

Keywords: COVID-19, Mental health-Propensity score matching, Pandemic.

\section{INTRODUCTION}

In late 2019 COVID-19 was at first rumored by China and had unfolded to thirteen countries by January

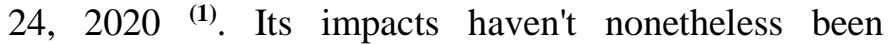
determined, as the observations and testing results are square measure dynamical quickly. Among the rife symptoms of this sickness, cough, fever, shortness of breath, and sometimes diarrhea. Older men with medical co-morbidities square measure a lot of doubtless to urge infected, with worse outcomes ${ }^{(2)}$. Severe cases will result in internal organ injury, respiratory failure, acute respiratory distress syndrome (ARDS), and death ${ }^{(3)}$.

COVID-19 epidemic is thought of as a general health crisis that has caused challenges for mental resilience and has been the most important natural event since the severe acute respiratory syndrome (SARS) outbreak in $2003^{(4)}$.

The planet has witnessed several widespread outbreaks of acute respiratory illnesses. For instance, SARS as a communicable infectious disease spread in 2003; however, it had been mostly managed by quarantine measures. However, the results of quarantine haven't been investigated ${ }^{(5)}$. The implications of the disease outbreaks touching all aspects of humans' lives have continued ${ }^{\left({ }^{(6)} \text {. }\right.}$

Mental health is one of the largest issues that have to be self-addressed currently, and post-pandemic as this crisis has generated tremendous stress publicly ${ }^{(7)}$.

The advent of COV-SARS has confused, modified people's living conditions, as well as commutation restrictions, the worry of disease transmission, and closure of schools and businesses ${ }^{(5)}$. In addition to specific features like its mode of transmission, rapidity of spread, and lack of definitive treatment protocols or vaccines all are responsible for the mental state issues ${ }^{(\mathbf{( 8 )}}$.

Although extensive restrictions, like the prohibition of national and international travel that reflects a replacement variety of quarantine area units effective in controlling and managing the disease, they need negative psychological impacts on individuals. Anxiety concerning food shortages is additional wide. Moreover, spreading rumors has negative psychological impacts ${ }^{(\boldsymbol{9})}$.

Psychiatric morbidities are found to vary from anxiety, depression, panic attacks, somatic symptoms, posttraumatic stress disorder, delirium, psychosis, and even suicidality ${ }^{(\mathbf{1 0})}$. Past tragedies have shown that the psychological state implications will last longer and have a bigger prevalence than the epidemic itself which the psychosocial and economic impacts are determinable if we tend to take into account their resonance in numerous contexts. (11). Therefore, psychiatric interventions are essential throughout infectious disease outbreaks with a high mortality rate ${ }^{(\mathbf{1 2})}$.

COV-SARS pandemic might endanger the mental well-being of the healthcare workers, inflicting depression, anxiety, insomnia, or distress. A sustained rise in cases, excessive employment, inevitable media coverage, and inadequate personal protecting instrumentality and medications were among the varied factors for the deterioration of the psychological state. $\mathrm{HCW}$ aren't solely petrified of obtaining infected however additionally upset concerning contaminating their families, friends, and colleagues with the virus ${ }^{(\mathbf{1 3})}$. 
During pandemics, it's common for health professionals, scientists, and managers to focus preponderantly on the microorganism and therefore the biological risk in an endeavor to grasp the pathophysiological mechanisms concerned and propose measures for preventing, containing, and treating the illness. In such situations, the psychological and psychiatric implications secondary to the phenomenon, both on an individual and a collective level, tend to be underestimated and neglected, generating gaps in cope ways and increasing the burden of associated diseases ${ }^{(14)}$.

This study aimed to outline some mental issues faced by HCW at the time of COV-SARS. The objectives of this study are to seek out the relationships between operating in medical institutions and mental health issues.

\section{PATIENTS AND METHOD}

A comparative cross-sectional study was conducted on 5745 participants in the Qalyubiya Governorate. As COV-SARS is a pandemic disease so detecting its prevalence or incidence is difficult nowadays. The estimated population of the Qaliubeya governorate was $5,745,000$, so studying $10 \%$ of the population at risk means choosing 5745 participants for the study. The Qaliubeya governorate includes 7 centers Benha $(167,029)$, Qalube $(100,495)$, Touch $(67,599)$, El-khanka $(62,434)$, Shibin El-kanater $(56,872)$, Elkanater Elkhairia $(56,302)$ and Kafr Shokr $(638,000)$. So sample will include 1670 from Benha, 1005 from Qalube, 676 from Touch, 624 from Elkhanka, 569 from ShibinElkanater, 563 from Elkanater Elkhairia, and 638 from Kafr Shokr. The answers of participants were chosen randomly from the total respondents.

Data collection: Data collection was carried out from March 22 to the end of May 2020 using the following Arabic online questionnaire sheet. It consisted of two parts, the first part assessed the socio-demographic characters of the participants. The second included the questions of the Fear of Coronavirus-19 Scale ${ }^{(\mathbf{1 5})}$ and The Hospital Anxiety and Depression Scale (HADS ${ }^{(16)}$ who translated and validated the items of the Iranian version.

The scale of COVID-19 fear (7 Qs scale) where the participants choose their level of agreement with the statements using a five-item Likert-type scale (strongly disagree, disagree, neither agree nor disagree, agree and strongly agree). The minimum score for each question is 1 , and the maximum is 5 (total is ranging from 7 to 35). The higher the score, the greater the fear of COVID-19.

HADS contains 14 items and consists of two subscales (anxiety and depression). Each item is rated on a four-point scale, giving maximum scores for anxiety and depression that equals 21 (Scores of $\geq 11=\mathrm{a}$ significant cause of psychological morbidity, $8-10=$ borderline, and 0-7 =normal).

\section{Ethical Considerations}

This study was approved by the Research Ethics Committee of the Benha University (number of RC 26-2020). Finally, informed online consent was obtained from all participants. It included data about the title, objectives, methods, benefits, and expected risks, and confidentiality of data. This work has been carried out following The Code of Ethics of the World Medical Association (Declaration of Helsinki) for studies involving humans.

\section{Data analysis:}

The collected data were tabulated and analyzed using the computer programs Statistical Package for Social Science (SPSS) version 25.0 for windows, (SPSS Inc, Chicago, IL). The collected data were summarized after establishing their non -normality by K-S test (OneSample Kolmogorov-Smirnov Test) of normality, in terms of median, inter-quartile range (IQR) for quantitative data, and frequency and proportion for qualitative data. Statistical comparisons between the different study groups were carried out using univariate tests including the Chi-square test $(\chi 2), \mathrm{Z}$ test of proportion, Kruskal- Wallis, Wilcoxon, and ManWhitney test as appropriate. Spearman correlation test and multiple linear regression analysis of fear, anxiety, or depression scores were conducted based on the results from the univariate correlation analysis. A P-value $<0.05$ was considered statistically significant. A pilot study was performed on $10 \%$ of participants and its results weren't included in the study.

Participants were divided according to their occupation into health care workers (HCWs) (physicians, nurses, technicians, and workers) and non-HCW and after comparing them, each group was studied separately to find the relationship between fear, anxiety, and depression scores, and other variables.

To reduce selection bias between both groups, propensity score matching (PSM) was used which matches participants of HCW with participants of non$\mathrm{HCW}$. Matching on propensity scores is performed through the use of a "greedy matching algorithm that sorts the observations in $\mathrm{HCW}$ group by their estimated propensity score and matches each unit separately to a unit in the non-HCW group that has the closest propensity score" The ratio of the propensity score variances in both groups was $0.9^{(17)}$.

\section{RESULTS}

This cross-sectional survey enrolled 5745 participants from the Qalyubia governorate aged from 18 to $>40$ years. Socio-demographic characteristics outlined that more than half of participants $(57.5 \%)$ were female, $96.9 \%$ were highly educated, $88.2 \%$ were from urban areas. Regarding anxiety level, $41.7 \%$ were considered as cases, while $26.6 \%$ suffered from depression.

Participants were divided according to their occupation into HCW(1740) and non-HCW (4005). By comparing both groups, Age, marital status, level of education, and residence were different significantly between them $(\mathrm{p}<0.001)$ (Table1).

After using PMS to minimize bias between the studied groups, $\mathrm{HCW}$ were prone to a considerable degree 
of mental illness due to the COVID-19 pandemic as they had significantly higher scores than non-HCW (fear median (IQR)(19.0 (15.0-22.0) versus 18.0(14.0-21.0), anxiety $\quad 11.0(7.0-12.0) \quad$ versus $9.0(6.0-11.0)$ and depression 9.0(7.0-11.0) versus 8.0(6.010.0)respectively) (Figure1): Box-plot of fear, anxiety and depression scores of HCW and non HCW.

Table 2 showed that socio demographic factors were associated with mental problems among $\mathrm{HCW}$ as female had significantly higher fear score (19.0 (15.0$22.0)$ ), anxiety score (11.0 (8.0-12.0)) and depression score $(9.0(8.0-11.0))$. HCW at the age group from 26 to 30 years had significantly higher scores of fear (20.0 (16.023.0)), anxiety (11.0 (10.0-12.0)) and depression (9.0 $(8.0-11.0))(\mathrm{p}<.001)$. Middle educated HCW participants had significant higher depression score (11.0 (9.25-15.5)) than higher educated persons. Non-married HCW showed higher fear score $(20.0(15.0-21.0))$ than non-married which was statistically significant $(\mathrm{P}=0.009)$. More than 4h of watching media about COVID-19 had significant higher fear (20.0 (15.0-25.0)), anxiety (11.0 (10.0-13.0)) and depression (10.0 (8.0-13.0)).

Regarding non-married groups among non-HCWs, there were statistically significant differences in scores of fear (18.0(15.0-21.0)) and anxiety (9.0 (6.0-11.0)). Low level of education had significant lower scores of fear $(12.0(11.0-12.5))(\mathrm{P}=0.018)$ More than $4 \mathrm{~h}$ of watching media about COVID-19 had significant higher fear (20.0 (16.0-23.0)), anxiety (11.0 (5.5-12.0)) and depression (9.0 (7.0-12.0))(Table 1).

Linear regression analysis among $\mathrm{HCW}$ showed that increase hours of watching media is a predictor of fear (95\% CI: 0.555-1.4, p<.001), anxiety (95\% CI: 0.77$1.22, \mathrm{p}<.001$ ), and depression (95\%CI: $0.45-0.85$, $\mathrm{p}<.001)$ and also among non-HCW (fear $(95 \% \mathrm{CI}$ : 1.44$2.2, \mathrm{p}<.001)$, anxiety (95\%CI: 0.94-1.49, $\mathrm{p}<.001)$. Depression core was directly correlated with increasing hours of watching media (rho $(\mathrm{P})$ : $0.106(<0.001))$ (Table 2).

Table (1): Comparison between health care workers and Non-HCW before and after PSM

\begin{tabular}{|c|c|c|c|c|c|c|c|c|c|c|c|c|c|c|}
\hline & \multicolumn{7}{|c|}{ Before PSM } & \multicolumn{7}{|c|}{ After PSM } \\
\hline & \multicolumn{2}{|c|}{$\begin{array}{c}\text { Total } \\
(\mathbf{5 7 4 5})\end{array}$} & \multicolumn{2}{|c|}{$\begin{array}{l}\text { HCW } \\
(1740)\end{array}$} & \multicolumn{2}{|c|}{$\begin{array}{c}\text { Non-HCW } \\
(4005)\end{array}$} & \multirow[t]{2}{*}{$\begin{array}{c}\mathbf{P} \\
\text { value }\end{array}$} & \multicolumn{2}{|c|}{$\begin{array}{c}\text { Total } \\
(\mathbf{3 4 8 0})\end{array}$} & \multicolumn{2}{|c|}{$\begin{array}{l}\text { HCW } \\
(1740)\end{array}$} & \multicolumn{2}{|c|}{$\begin{array}{c}\text { Non-HCW } \\
(\mathbf{1 7 4 0 )} \\
\end{array}$} & \multirow[t]{2}{*}{$\begin{array}{c}\text { P- } \\
\text { value }\end{array}$} \\
\hline & No & $\%$ & No & $\%$ & No & $\%$ & & No & $\%$ & No & $\%$ & No & $\%$ & \\
\hline $\begin{array}{l}\text { Gender } \\
\text { Male } \\
\text { Female }\end{array}$ & $\begin{array}{l}2439 \\
3306 \\
\end{array}$ & $\begin{array}{l}42.5 \\
57.5\end{array}$ & $\begin{array}{l}730 \\
1010 \\
\end{array}$ & $\begin{array}{l}42.0 \\
58.0\end{array}$ & $\begin{array}{l}1709 \\
2296 \\
\end{array}$ & $\begin{array}{l}42.7 \\
57.3 \\
\end{array}$ & 0.61 & $\begin{array}{l}1477 \\
2003\end{array}$ & $\begin{array}{l}42.4 \\
57.6 \\
\end{array}$ & $\begin{array}{l}730 \\
1010\end{array}$ & $\begin{array}{l}42.0 \\
58.0\end{array}$ & $\begin{array}{l}747 \\
993\end{array}$ & $\begin{array}{l}42.9 \\
57.1\end{array}$ & 0.56 \\
\hline $\begin{array}{l}\text { Age } \\
18 y- \\
26 y- \\
31 y- \\
>40\end{array}$ & $\begin{array}{l}1890 \\
1121 \\
1808 \\
926 \\
\end{array}$ & $\begin{array}{l}32.9 \\
19.5 \\
31.5 \\
16.1\end{array}$ & $\begin{array}{l}91 \\
312 \\
873 \\
464\end{array}$ & $\begin{array}{l}5.2 \\
17.9 \\
50.2 \\
26.7\end{array}$ & $\begin{array}{l}1799 \\
809 \\
935 \\
462 \\
\end{array}$ & $\begin{array}{l}44.9 \\
20.2 \\
23.3 \\
11.5\end{array}$ & $\begin{array}{l}<0.001 \\
0.043 \\
<0.001 \\
<0.001 \\
\end{array}$ & $\begin{array}{l}188 \\
636 \\
1754 \\
902 \\
\end{array}$ & $\begin{array}{l}5.4 \\
18.3 \\
50.4 \\
25.9 \\
\end{array}$ & $\begin{array}{l}91 \\
312 \\
873 \\
464\end{array}$ & $\begin{array}{l}5.2 \\
17.9 \\
50.2 \\
26.7 \\
\end{array}$ & $\begin{array}{l}97 \\
324 \\
881 \\
438 \\
\end{array}$ & $\begin{array}{l}5.6 \\
18.6 \\
50.6 \\
25.2 \\
\end{array}$ & 0.75 \\
\hline $\begin{array}{l}\text { Marital status } \\
\text { Married } \\
\text { Not } \\
\end{array}$ & $\begin{array}{l}2942 \\
2803 \\
\end{array}$ & $\begin{array}{l}51.2 \\
48.8\end{array}$ & $\begin{array}{l}1446 \\
294 \\
\end{array}$ & $\begin{array}{l}83.1 \\
16.9 \\
\end{array}$ & $\begin{array}{l}1496 \\
2509 \\
\end{array}$ & $\begin{array}{l}37.4 \\
62.6 \\
\end{array}$ & $<0.001$ & $\begin{array}{l}2862 \\
618 \\
\end{array}$ & $\begin{array}{l}82.2 \\
17.8\end{array}$ & $\begin{array}{l}1446 \\
294 \\
\end{array}$ & $\begin{array}{l}83.1 \\
16.9 \\
\end{array}$ & $\begin{array}{l}1416 \\
324\end{array}$ & $\begin{array}{l}81.4 \\
18.6 \\
\end{array}$ & 0.183 \\
\hline $\begin{array}{l}\text { Level of } \\
\text { education } \\
\text { Read \&write } \\
\text { Middle } \\
\text { High } \\
\end{array}$ & $\begin{array}{l}30 \\
147 \\
5568\end{array}$ & $\begin{array}{l}0.5 \\
2.6 \\
96.9\end{array}$ & $\begin{array}{l}0 \\
20 \\
1720\end{array}$ & $\begin{array}{l}0.0 \\
1.1 \\
98.9\end{array}$ & $\begin{array}{l}30 \\
127 \\
3848\end{array}$ & $\begin{array}{l}0.7 \\
3.2 \\
96.1\end{array}$ & $<0.001$ & $\begin{array}{l}4 \\
45 \\
3431\end{array}$ & $\begin{array}{l}0.1 \\
1.3 \\
98.6\end{array}$ & $\begin{array}{l}0 \\
20 \\
1720\end{array}$ & $\begin{array}{l}0.0 \\
1.1 \\
98.9\end{array}$ & $\begin{array}{l}4 \\
25 \\
1711\end{array}$ & $\begin{array}{l}0.2 \\
1.4 \\
98.3\end{array}$ & 0.101 \\
\hline $\begin{array}{l}\text { Residence } \\
\text { Urban } \\
\text { Rural }\end{array}$ & $\begin{array}{l}5066 \\
679 \\
\end{array}$ & $\begin{array}{l}88.2 \\
11.8 \\
\end{array}$ & $\begin{array}{l}1391 \\
349 \\
\end{array}$ & $\begin{array}{l}79.9 \\
20.1 \\
\end{array}$ & $\begin{array}{l}3675 \\
330 \\
\end{array}$ & $\begin{array}{l}91.8 \\
8.2 \\
\end{array}$ & $<0.001$ & $\begin{array}{l}2809 \\
671 \\
\end{array}$ & $\begin{array}{l}80.7 \\
19.3 \\
\end{array}$ & $\begin{array}{l}1391 \\
349 \\
\end{array}$ & $\begin{array}{l}79.9 \\
20.1 \\
\end{array}$ & $\begin{array}{l}1418 \\
322\end{array}$ & $\begin{array}{l}81.5 \\
18.5 \\
\end{array}$ & 0.246 \\
\hline $\begin{array}{l}\text { Centers of } \\
\text { residence } \\
\text { Benha } \\
\text { Qalube } \\
\text { Toukh } \\
\text { El-Khanka } \\
\text { Shibin } \\
\text { Elkanater } \\
\text { Elkanater- } \\
\text { Elkhairia } \\
\text { Kafr Shokr }\end{array}$ & $\begin{array}{l}1670 \\
1005 \\
676 \\
624 \\
569 \\
563 \\
638\end{array}$ & $\begin{array}{l}29.1 \\
17.5 \\
11.8 \\
10.9 \\
9.9 \\
9.8 \\
11.1\end{array}$ & $\begin{array}{l}505 \\
264 \\
247 \\
224 \\
77 \\
211 \\
212\end{array}$ & $\begin{array}{l}29.0 \\
15.2 \\
14.2 \\
12.9 \\
4.4 \\
12.1 \\
12.2\end{array}$ & $\begin{array}{l}1165 \\
741 \\
429 \\
400 \\
492 \\
352 \\
426\end{array}$ & $\begin{array}{l}29.1 \\
18.5 \\
10.7 \\
10.0 \\
12.3 \\
8.8 \\
10.6\end{array}$ & $\begin{array}{l}0.94 \\
0.003 \\
<0.001 \\
0.001 \\
<0.001 \\
<0.001 \\
0.08\end{array}$ & $\begin{array}{l}880 \\
497 \\
454 \\
344 \\
328 \\
425 \\
552\end{array}$ & $\begin{array}{l}25.3 \\
14.3 \\
13.0 \\
9.9 \\
9.4 \\
12.2 \\
15.9\end{array}$ & $\begin{array}{l}505 \\
264 \\
247 \\
224 \\
77 \\
211 \\
212\end{array}$ & $\begin{array}{l}29.0 \\
15.2 \\
14.2 \\
12.9 \\
4.4 \\
12.1 \\
12.2\end{array}$ & $\begin{array}{l}375 \\
233 \\
207 \\
120 \\
251 \\
214 \\
340\end{array}$ & $\begin{array}{l}21.6 \\
13.4 \\
11.9 \\
6.9 \\
14.4 \\
12.3 \\
19.5\end{array}$ & $<0.001$ \\
\hline $\begin{array}{l}\text { Hours of } \\
\text { watching } \\
\text { media } \\
<1 \mathrm{~h} \\
2-4 \mathrm{~h} \\
>4 \mathrm{~h}\end{array}$ & $\begin{array}{l}4006 \\
1212 \\
527\end{array}$ & $\begin{array}{l}69.7 \\
21.1 \\
9.2\end{array}$ & $\begin{array}{l}958 \\
543 \\
239\end{array}$ & $\begin{array}{l}55.1 \\
31.2 \\
13.7\end{array}$ & $\begin{array}{l}3048 \\
669 \\
288\end{array}$ & $\begin{array}{l}76.1 \\
16.7 \\
7.2\end{array}$ & $\begin{array}{l}<0.001 \\
<0.001 \\
<0.001\end{array}$ & $\begin{array}{l}2190 \\
915 \\
375\end{array}$ & $\begin{array}{l}62.9 \\
26.3 \\
10.8\end{array}$ & $\begin{array}{l}958 \\
543 \\
239\end{array}$ & $\begin{array}{l}55.1 \\
31.2 \\
13.7\end{array}$ & $\begin{array}{l}1232 \\
372 \\
136\end{array}$ & $\begin{array}{l}70.8 \\
21.4 \\
7.8\end{array}$ & $<0.001$ \\
\hline
\end{tabular}

PSM: propensity score matching P: probability 
The studied group

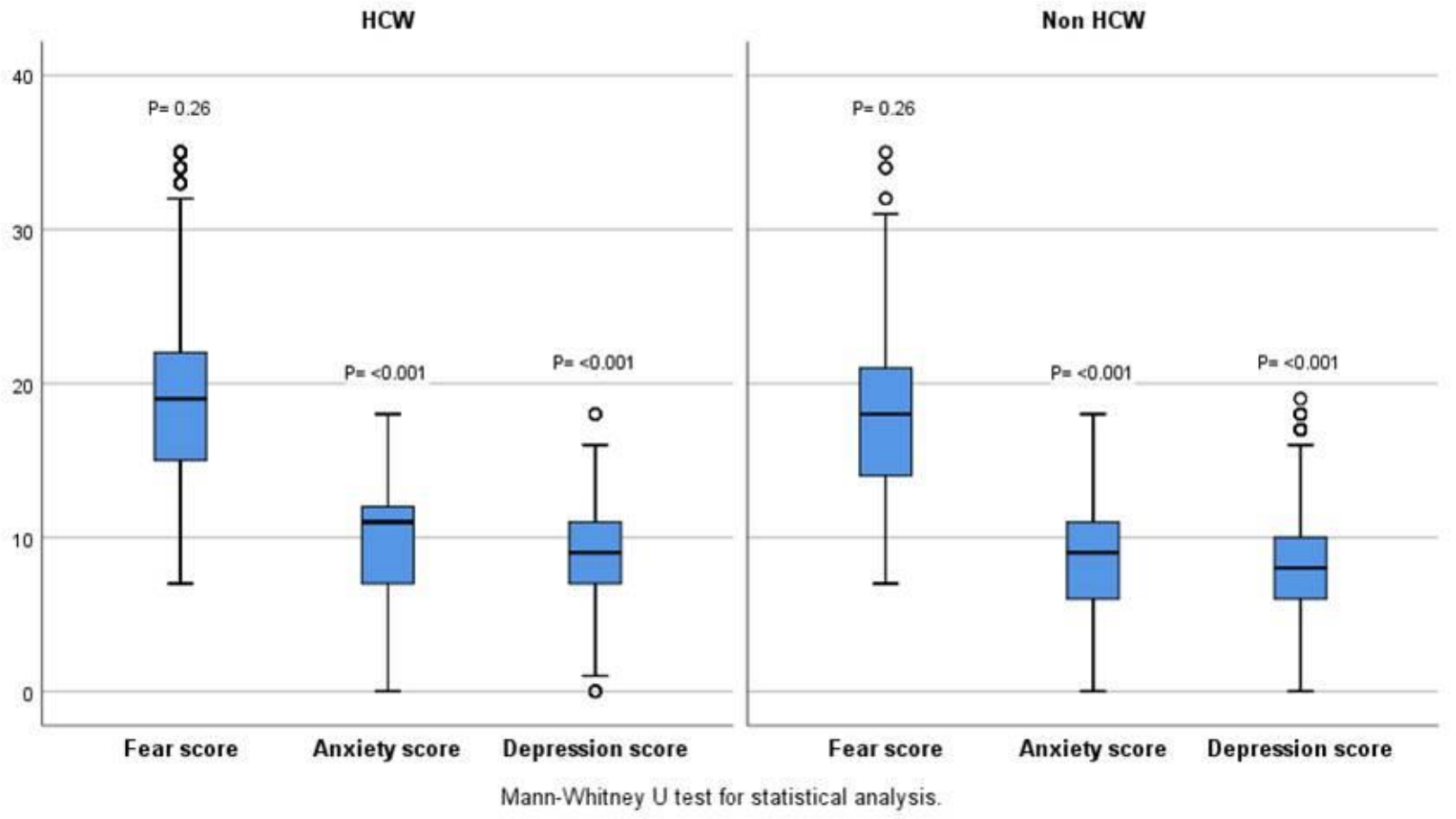

Figure (1): Box-plot of fear, anxiety, and depression scores of HCW and non HCW.

Table (2): Differences of fear, anxiety, and depression scores regarding other variables among HCW and non-HCW groups.

\begin{tabular}{|c|c|c|c|c|c|c|c|c|c|c|}
\hline & \multirow[t]{2}{*}{ No } & \multicolumn{2}{|c|}{ Fear score } & \multirow{2}{*}{$\begin{array}{c}\text { P- } \\
\text { value }\end{array}$} & \multicolumn{2}{|c|}{ Anxiety score } & \multirow{2}{*}{$\begin{array}{c}\text { P- } \\
\text { value }\end{array}$} & \multicolumn{2}{|c|}{ Dep score } & \multirow{2}{*}{$\begin{array}{c}\text { P- } \\
\text { value }\end{array}$} \\
\hline & & Median & IQR & & Median & IQR & & Median & IQR & \\
\hline \multicolumn{11}{|l|}{ HCW (1740) } \\
\hline $\begin{array}{l}\text { Gender } \\
\text { Male } \\
\text { Female }\end{array}$ & $\begin{array}{l}730 \\
1010\end{array}$ & $\begin{array}{l}18.0 \\
19.0\end{array}$ & $\begin{array}{l}14.0-22.0 \\
15.0-22.0\end{array}$ & 0.014 & $\begin{array}{l}10.0 \\
11.0\end{array}$ & $\begin{array}{l}6.75-12.0 \\
8.0-12.0\end{array}$ & $<0.001$ & $\begin{array}{l}9.0 \\
9.0\end{array}$ & $\begin{array}{l}7.0-11.0 \\
8.0-11.0\end{array}$ & 0.002 \\
\hline $\begin{array}{l}\text { Age } \\
18 y- \\
26 y- \\
31 y- \\
>40\end{array}$ & $\begin{array}{l}91 \\
312 \\
873 \\
464\end{array}$ & $\begin{array}{l}18.0 \\
20.0 \\
19.0 \\
18.0\end{array}$ & $\begin{array}{l}15.0-21.0 \\
16.0-23.0 \\
14.0-21.0 \\
15.0-21.0\end{array}$ & $<0.001$ & $\begin{array}{l}11.0 \\
11.0 \\
10.0 \\
10.0\end{array}$ & $\begin{array}{l}8.0-11.0 \\
10.0-12.0 \\
8.0-12.0 \\
7.0-12.0\end{array}$ & $<0.001$ & $\begin{array}{l}8.0 \\
9.0 \\
9.0 \\
9.0\end{array}$ & $\begin{array}{l}7.0-11.0 \\
8.0-12.0 \\
7.0-11.0 \\
7.0-10.0\end{array}$ & 0.003 \\
\hline $\begin{array}{l}\text { Marital status } \\
\text { Married } \\
\text { Not }\end{array}$ & $\begin{array}{l}1446 \\
294\end{array}$ & $\begin{array}{l}19.0 \\
20.0\end{array}$ & $\begin{array}{l}15.0-23.0 \\
15.0-24.0\end{array}$ & 0.009 & $\begin{array}{l}10.0 \\
10.0\end{array}$ & $\begin{array}{l}7.0-12.0 \\
7.0-12.0\end{array}$ & 0.122 & $\begin{array}{l}9.0 \\
9.0\end{array}$ & $\begin{array}{l}7.0-11.0 \\
8.0-11.0\end{array}$ & 0.218 \\
\hline $\begin{array}{l}\text { Level of } \\
\text { education } \\
\text { Middle } \\
\text { High }\end{array}$ & $\begin{array}{l}20 \\
1720\end{array}$ & $\begin{array}{l}20.0 \\
19.0\end{array}$ & $\begin{array}{l}15.0-21.0 \\
15.0-22.0\end{array}$ & 0.28 & $\begin{array}{l}9.0 \\
11.0\end{array}$ & $\begin{array}{l}5.0-10.0 \\
7.0-12.0\end{array}$ & 0.088 & $\begin{array}{l}11.0 \\
9.0\end{array}$ & $\begin{array}{l}9.25-15.5 \\
7.0-11.0\end{array}$ & 0.003 \\
\hline $\begin{array}{l}\text { Residence } \\
\text { Urban } \\
\text { Rural } \\
\end{array}$ & $\begin{array}{l}1391 \\
349 \\
\end{array}$ & $\begin{array}{l}18.0 \\
20.0 \\
\end{array}$ & $\begin{array}{l}15.0-22.0 \\
15.0-22.0\end{array}$ & 0.058 & $\begin{array}{l}11.0 \\
11.0 \\
\end{array}$ & $\begin{array}{l}7.0-12.0 \\
8.0-12.0\end{array}$ & 0.003 & $\begin{array}{l}9.0 \\
9.0\end{array}$ & $\begin{array}{l}7.0-11.0 \\
8.0-11.0\end{array}$ & 0.021 \\
\hline $\begin{array}{l}\text { Hours of watching } \\
\text { media } \\
<1 \mathrm{~h} \\
2-4 \mathrm{~h} \\
>4 \mathrm{~h}\end{array}$ & $\begin{array}{l}958 \\
543 \\
239\end{array}$ & $\begin{array}{l}18.0 \\
19.0 \\
20.0\end{array}$ & $\begin{array}{l}14.0-21.0 \\
17.0-21.0 \\
15.0-25.0\end{array}$ & $<0.001$ & $\begin{array}{l}10.0 \\
11.0 \\
11.0\end{array}$ & $\begin{array}{l}7.0-12.0 \\
7.0-12.0 \\
10.0-13.0\end{array}$ & $<0.001$ & $\begin{array}{l}9.0 \\
9.0 \\
10.0\end{array}$ & $\begin{array}{l}7.0-11.0 \\
8.0-11.0 \\
8.0-13.0\end{array}$ & $\begin{array}{l}<0.00 \\
1\end{array}$ \\
\hline \multicolumn{11}{|l|}{ Non-HCW (1740) } \\
\hline $\begin{array}{l}\text { Gender } \\
\text { Male } \\
\text { Female }\end{array}$ & $\begin{array}{l}747 \\
993\end{array}$ & $\begin{array}{l}18.0 \\
17.0\end{array}$ & $\begin{array}{l}15.0-21.0 \\
14.0-21.0\end{array}$ & 0.053 & $\begin{array}{l}9.0 \\
9.0\end{array}$ & $\begin{array}{l}6.0-11.0 \\
6.0-11.0\end{array}$ & 0.25 & $\begin{array}{l}8.0 \\
8.0\end{array}$ & $\begin{array}{l}6.0-10.0 \\
6.0-10.0\end{array}$ & 0.80 \\
\hline $\begin{array}{l}\text { Age } \\
18 y- \\
26 y- \\
31 y- \\
>40\end{array}$ & $\begin{array}{l}97 \\
324 \\
881 \\
438\end{array}$ & $\begin{array}{l}18.0 \\
18.0 \\
18.0 \\
18.0\end{array}$ & $\begin{array}{l}15.0-21.0 \\
15.0-21.0 \\
14.0-21.0 \\
14.0-22.0\end{array}$ & 0.47 & $\begin{array}{l}8.0 \\
9.0 \\
9.0 \\
9.0\end{array}$ & $\begin{array}{l}6.0-10.5 \\
6.0-11.0 \\
6.0-11.0 \\
6.0-11.0\end{array}$ & 0.72 & $\begin{array}{l}8.0 \\
8.0 \\
8.0 \\
8.0\end{array}$ & $\begin{array}{l}6.0-10.0 \\
6.0-10.0 \\
6.0-10.0 \\
6.0-10.0\end{array}$ & 0.80 \\
\hline
\end{tabular}


https://ejhm.journals.ekb.eg/

\begin{tabular}{|c|c|c|c|c|c|c|c|c|c|c|}
\hline & \multirow[t]{2}{*}{ No } & \multicolumn{2}{|c|}{ Fear score } & \multirow{2}{*}{$\begin{array}{c}\text { P- } \\
\text { value }\end{array}$} & \multicolumn{2}{|c|}{ Anxiety score } & \multirow{2}{*}{$\begin{array}{c}\text { P- } \\
\text { value }\end{array}$} & \multicolumn{2}{|c|}{ Dep score } & \multirow{2}{*}{$\begin{array}{c}\text { P- } \\
\text { value }\end{array}$} \\
\hline & & Median & IQR & & Median & IQR & & Median & IQR & \\
\hline HCW (1740) & & & & & & & & & & \\
\hline $\begin{array}{l}\text { Gender } \\
\text { Male } \\
\text { Female }\end{array}$ & $\begin{array}{l}730 \\
1010\end{array}$ & $\begin{array}{l}18.0 \\
19.0\end{array}$ & $\begin{array}{l}14.0-22.0 \\
15.0-22.0\end{array}$ & 0.014 & $\begin{array}{l}10.0 \\
11.0\end{array}$ & $\begin{array}{l}6.75-12.0 \\
8.0-12.0\end{array}$ & $<0.001$ & $\begin{array}{l}9.0 \\
9.0\end{array}$ & \begin{tabular}{|l|}
$7.0-11.0$ \\
$8.0-11.0$
\end{tabular} & 0.002 \\
\hline $\begin{array}{l}\text { Age } \\
18 \mathrm{y}- \\
26 \mathrm{y}- \\
31 \mathrm{y}- \\
>40\end{array}$ & $\begin{array}{l}91 \\
312 \\
873 \\
464 \\
\end{array}$ & $\begin{array}{l}18.0 \\
20.0 \\
19.0 \\
18.0\end{array}$ & $\begin{array}{l}15.0-21.0 \\
16.0-23.0 \\
14.0-21.0 \\
15.0-21.0\end{array}$ & $<0.001$ & $\begin{array}{l}11.0 \\
11.0 \\
10.0 \\
10.0\end{array}$ & $\begin{array}{l}8.0-11.0 \\
10.0-12.0 \\
8.0-12.0 \\
7.0-12.0\end{array}$ & $<0.001$ & $\begin{array}{l}8.0 \\
9.0 \\
9.0 \\
9.0\end{array}$ & \begin{tabular}{|l|}
$7.0-11.0$ \\
$8.0-12.0$ \\
$7.0-11.0$ \\
$7.0-10.0$ \\
\end{tabular} & 0.003 \\
\hline $\begin{array}{l}\text { Marital status } \\
\text { Married } \\
\text { Not }\end{array}$ & $\begin{array}{l}1446 \\
294\end{array}$ & $\begin{array}{l}19.0 \\
20.0\end{array}$ & $\begin{array}{l}15.0-23.0 \\
15.0-24.0\end{array}$ & 0.009 & $\begin{array}{l}10.0 \\
10.0\end{array}$ & $\begin{array}{l}7.0-12.0 \\
7.0-12.0\end{array}$ & 0.122 & $\begin{array}{l}9.0 \\
9.0\end{array}$ & $\begin{array}{l}7.0-11.0 \\
8.0-11.0\end{array}$ & 0.218 \\
\hline $\begin{array}{l}\text { Marital status } \\
\text { Married } \\
\text { Not }\end{array}$ & $\begin{array}{l}1416 \\
324\end{array}$ & $\begin{array}{l}17.0 \\
18.0\end{array}$ & $\begin{array}{l}13.0-21.0 \\
15.0-21.0\end{array}$ & 0.006 & $\begin{array}{l}8.0 \\
9.0\end{array}$ & $\begin{array}{l}5.0-11.0 \\
6.0-11.0\end{array}$ & 0.003 & $\begin{array}{l}8.0 \\
8.0\end{array}$ & $\begin{array}{l}6.0-10.0 \\
6.0-10.0\end{array}$ & 0.535 \\
\hline $\begin{array}{l}\text { Level of education } \\
\text { Read \&write } \\
\text { Middle } \\
\text { High } \\
\end{array}$ & $\begin{array}{l}4 \\
25 \\
1711 \\
\end{array}$ & $\begin{array}{l}12.0 \\
20.0 \\
18.0\end{array}$ & $\begin{array}{l}11.0-12.5 \\
15.0-22.5 \\
15.0-21.0 \\
\end{array}$ & 0.018 & $\begin{array}{l}7.0 \\
8.0 \\
9.0 \\
\end{array}$ & $\begin{array}{l}5.0-9.0 \\
5.5-10.5 \\
6.0-11.0 \\
\end{array}$ & 0.50 & $\begin{array}{l}6.0 \\
9.0 \\
8.0\end{array}$ & $\begin{array}{l}6.0-6.0 \\
7.0-11.5 \\
6.0-10.0 \\
\end{array}$ & 0.10 \\
\hline $\begin{array}{l}\text { Residence } \\
\text { Urban } \\
\text { Rural }\end{array}$ & $\begin{array}{l}1418 \\
322\end{array}$ & $\begin{array}{l}18.0 \\
18.0 \\
\end{array}$ & $\begin{array}{l}15.0-21.0 \\
13.0-21.0 \\
\end{array}$ & 0.098 & $\begin{array}{l}9.0 \\
9.0 \\
\end{array}$ & $\begin{array}{l}6.0-11.0 \\
6.0-11.0 \\
\end{array}$ & 0.37 & $\begin{array}{l}8.0 \\
8.0 \\
\end{array}$ & $\begin{array}{l}6.0-10.0 \\
6.0-11.0 \\
\end{array}$ & 0.57 \\
\hline $\begin{array}{l}\text { Hours of watching } \\
\text { media } \\
<1 \mathrm{~h} \\
2-4 \mathrm{~h} \\
>4 \mathrm{~h}\end{array}$ & $\begin{array}{l}1232 \\
372 \\
136\end{array}$ & $\begin{array}{l}17.0 \\
18.5 \\
20.0\end{array}$ & $\begin{array}{l}14.0-20.0 \\
15.0-23.0 \\
16.0-23.0\end{array}$ & $<0.001$ & $\begin{array}{l}8.0 \\
10.0 \\
11.0\end{array}$ & $\begin{array}{l}6.0-11.0 \\
8.0-12.0 \\
5.25- \\
12.0 \\
\end{array}$ & $<0.001$ & $\begin{array}{l}8.0 \\
8.5 \\
9.0\end{array}$ & $\begin{array}{l}6.0-10.0 \\
7.0-11.0 \\
7.0-12.0\end{array}$ & $<0.001$ \\
\hline
\end{tabular}

P: probability

Table (3): Multivariate linear regression of different scores among HCW and non-HCW groups.

\begin{tabular}{|c|c|c|c|c|c|c|c|c|c|c|c|c|c|c|c|}
\hline & \multicolumn{5}{|c|}{ Fear scores } & \multicolumn{5}{|c|}{ Anxiety scores } & \multicolumn{5}{|c|}{ Depression scores } \\
\hline & \multicolumn{2}{|c|}{ Univariate } & \multicolumn{3}{|c|}{ Multivariate } & \multicolumn{2}{|c|}{ Univariate } & \multicolumn{3}{|c|}{ Multivariate } & \multicolumn{2}{|c|}{ Univariate } & \multicolumn{3}{|c|}{ Multivariate } \\
\hline & rho & p & B & $\mathbf{P}$ & $95 \% \mathrm{CI}$ & rho & p & B & $\mathbf{P}$ & $95 \% \mathrm{CI}$ & rho & $\mathbf{p}$ & B & $\mathbf{P}$ & 95\%CI \\
\hline \multicolumn{16}{|l|}{ HCW } \\
\hline $\begin{array}{l}\text { Gender } \\
\text { (male:0) }\end{array}$ & 0.059 & 0.014 & 0.117 & 0.712 & $-0.5-0.74$ & 0.115 & $<0.001$ & 0.838 & $<0.001$ & $0.51-1.17$ & 0.074 & 0.002 & 0.26 & 0.08 & $\begin{array}{l}(-0.03)- \\
(0.55)\end{array}$ \\
\hline Age & -0.086 & $<0.001$ & -0.989 & $<0.001$ & $\begin{array}{l}(-1.4)- \\
(-0.58)\end{array}$ & -0.147 & $<0.001$ & -0.591 & $<0.001$ & \begin{tabular}{|l}
$(-0.80)-(-$ \\
$0.39)$
\end{tabular} & -0.068 & 0.005 & -0.301 & 0.001 & \begin{tabular}{|l|}
$(-0.48)-(-$ \\
$0.12)$
\end{tabular} \\
\hline $\begin{array}{l}\text { Marital } \\
\text { status } \\
\text { (unmarried: } \\
\text { 0) }\end{array}$ & 0.063 & 0.009 & 1.66 & $<0.001$ & $0.768-2.5$ & -0.037 & 0.122 & & & & & & & & \\
\hline $\begin{array}{l}\text { Residence } \\
\text { (rural:0) }\end{array}$ & -0.045 & 0.058 & & & & -0.07 & 0.003 & -0.568 & 0.006 & \begin{tabular}{|l|}
$(-0.98)-(-$ \\
$0.16)$
\end{tabular} & -0.055 & 0.021 & -1.42 & 0.62 & $\begin{array}{l}(-0.62)- \\
(0.098)\end{array}$ \\
\hline $\begin{array}{l}\text { Education } \\
\text { level }\end{array}$ & -0.026 & 0.282 & -1.08 & 0.451 & $-3.87-1.720$ & 20.041 & 0.088 & & & & -0.072 & 0.003 & -2.16 & 0.001 & \begin{tabular}{|l|}
$(-3.47)-(-$ \\
$0.85)$
\end{tabular} \\
\hline $\begin{array}{l}\text { Hours of } \\
\text { watching } \\
\text { media }\end{array}$ & 0.118 & $<0.001$ & 0.979 & $<0.001$ & $0.555-1.4$ & 0.18 & $<0.001$ & 0.993 & $<0.001$ & $0.77-1.22$ & 0.131 & $<0.001$ & 0.65 & $<0.001$ & $0.45-0.85$ \\
\hline $\mathrm{r}^{2}\left(\operatorname{adj} \mathrm{r}^{2}\right)$ & & & \multicolumn{3}{|c|}{$0.024(0.022)$} & & & \multicolumn{3}{|c|}{$0.077(0.074)$} & & & \multicolumn{3}{|c|}{$\frac{1}{0.04(0.037)}$} \\
\hline $\mathrm{F}(\mathrm{p})$ & & & \multicolumn{3}{|c|}{$8.7(<0.001)$} & & & \multicolumn{3}{|c|}{$35.94(<0.001)$} & & & \multicolumn{3}{|c|}{$14.41(<0.001)$} \\
\hline \multicolumn{16}{|l|}{ Non-HCW } \\
\hline $\begin{array}{l}\text { Marital } \\
\text { status } \\
\text { (unmarried: } \\
\text { 0) }\end{array}$ & 0.063 & 0.004 & 0.79 & 0.011 & $0.18-1.3$ & 0.075 & 0.001 & 0.70 & 0.002 & $0.25-1.1$ & & & & & \\
\hline $\begin{array}{l}\text { Education } \\
\text { level }\end{array}$ & 0.007 & 0.38 & 0.19 & 0.81 & $\begin{array}{l}-1.36- \\
1.73\end{array}$ & & & & & & & & & & \\
\hline $\begin{array}{l}\text { Hours of } \\
\text { watching } \\
\text { media }\end{array}$ & 0.222 & $<0.001$ & 1.82 & $<0.001$ & $1.44-2.2$ & 0.20 & $<0.001$ & 1.21 & $<0.001$ & $0.94-1.4$ & 0.106 & $<0.001$ & & & \\
\hline $\mathrm{r}^{2}\left(\operatorname{adjr}^{2}\right)$ & & & \multirow{2}{*}{\multicolumn{3}{|c|}{$\frac{0.053(0.051)}{3237(<0001)}$}} & & & \multicolumn{3}{|c|}{$0.046(0.045)$} & & & & & \\
\hline $\mathrm{F}(\mathrm{p})$ & & & & & & & & 41.76 & $6(<0.001)$ & & & & & & \\
\hline
\end{tabular}

CI: confidence interval rho: spearman correlation test p: probability 


\section{DISCUSSION}

This cross-sectional survey listed 5745 participants from the seven cities of the Qalyubia governorate. Egypt. Socio-demographic characteristics unconcealed that more than half of participants (57.5\%) were female, the majority were highly educated(96.9\%) and from urban areas(88.2\%) belonged to age groups ranging from 18 to $>40$ years. these findings correspond with a systematic review of studies conducted on Mental health problems faced by $\mathrm{HCW}$ due to the COVID-19 pandemic. In which 6 original articles were included and showed that the predominant participants in 4 studies were females $(68.7 \%-85.5 \%)^{(\mathbf{1 8})}$ and nearly all participants $(97.1 \%)$ lived in urban areas ${ }^{(\mathbf{1 3})}$.

Regarding anxiety level, $41.7 \%$ of all the study participants were thought about as cases. While $26.6 \%$ suffered from depression. These results square measure more than a study of psychological responses and associated factors during the Initial Stage of COVID19. The epidemic among the general population in China wherever ${ }^{(4)}$ this distinction could also be because of cultural and awareness variability across countries.

The current study suggests that HCW square measures are susceptible to a substantial degree of psychopathy because of the COVID-19 pandemic. the results showed that the median (IQR) scores of fear, anxiety, and depression were statistically considerably higher among HCW than non-HCW $((19.0(15.0-22.0)$ vs $18.0(14.0-21.0), \quad 11.0(7.0-12.0)$ versus $9.0(6.0-$ 11.0)and9.0(7.0-11.0)versus $8.0(6.0-$ 10.0)respectively).it could be explained by Workrelated stress with multiple clinical activities, depression in the face of the coexistence of countless deaths, long work shifts with the most diverse unknowns, and demands in the treatment with patients with COVID-19 ${ }^{(19)}$.

These findings are supported by another study (20) that stated that $\mathrm{HCW}$ is at significant risk of adverse mental health outcomes during the COVID-19 outbreak. And the rate of distress among healthcare staff is higher compared with the general population because they are more at risk for infection and transmission ${ }^{(21)}$.

Other previous studies reported that $\mathrm{HCW}$ especially those working in emergency units, intensive care units, and infectious disease wards are at higher risk of developing adverse psychiatric impact ${ }^{(22)}$.

Also, and it was established that the Corona virus generates a lot of uncertainty, and this has a particular resonance with health professionals who suffer or have suffered from anxiety, obsessive-compulsive disorder, and OCD in the treatment of patients in hospitals. Panic attacks can also be a response to the stress load linked to the demands of the Corona virus outbreak ${ }^{(\mathbf{1 9})}$.

Regarding socio-demographic factors associated with mental problems among HCW in this study female had higher scores (median (IQR)) than male regarding (fear (19.0 (15.0-22.0)) P =.014, anxiety (11.0 (8.012.0)) $\mathrm{P}<.001$ and depression $(9.0(8.0-11.0)) \mathrm{p}=.002)$. Linear regression analysis showed that being female is a predictor for anxiety $(95 \% \mathrm{CI}, 0.51-1.17 ; \mathrm{P}<.001)$ this is similar to ${ }^{(23)}$ where the score of depression in female medical staff $(27.08 \pm 4.6$ vs. $25.33 \pm 4.3, \mathrm{P}=0.011)$ was higher than that in the male). Also, Lai et al. ${ }^{(3)}$ showed that being a woman and possessing an intermediate professional title was associated with higher anxiety, depression, and distress.

This study showed that HCW at the age group from 26 to 30 years had significantly higher scores of fear (20.0 (16.0-23.0)), anxiety (11.0 (10.0-12.0)) and depression (9.0 (8.0-11.0)) ( $\mathrm{p}<.001)$. This corresponds to another study ${ }^{(24)}$ where medical staff at a younger age ( $<30$ years) had higher self-rated depression scores than those with older age (30 years).

Regarding educational level, this study found that Middle educated HCW participants had significantly higher depression scores (11.0 (9.25-15.5) than higher educated persons $(9.0(7.0-11.0)) \mathrm{p}=.003$ which is in line with the results that explained this as a higher level of education will improve one's understanding of issues associated with psychological distress, and increasing confidence in mental health recovery ${ }^{(25)}$.

In terms of marital status, Non- Married HCW showed a higher fear score median (IQR) (20.0 (15.021.0) $\mathrm{p}$.009) than married ., also the unmarried nonHCWs participants had significantly higher fear score median (IQR)( 18.0(15.0-21.0) $\mathrm{P}=0.006)$ and anxiety score median $(\mathrm{IQR})(9.0(6.0-11.0) \mathrm{p}=0.003)$ than married this was supported by results which stated that Married physicians reported less stress than nonmarried ones, it could be explained by family financial loss, a lack of support against negative emotions or depression symptoms ${ }^{(26)}$.

Linear regression analysis among $\mathrm{HCW}$ showed that increase hours of watching media is a predictor of fear (95\%CI: 0.555-1.4, p<.001), anxiety (95\%CI: $0.77-$ $1.22, \mathrm{p}<.001$ ), and depression (95\%CI: 0.45-0.85, $\mathrm{p}<.001)$ and also among non-HCW (fear $(95 \% \mathrm{CI}$ : 1.44$2.2, \mathrm{p}<.001)$, anxiety (95\%CI: 0.94-1.49, $\mathrm{p}<.001)$. Depression core was directly correlated with increasing hours of watching media (rho $(\mathrm{P}): 0.106(<0.001))$.so Minimizing watching, reading, or listening to news about COVID-19 that causes anxiety or distress is important for mental health ${ }^{(27)}$.

It was also evidenced by a cross-sectional study among Chinese citizens to assess the prevalence of mental health problems and examine their association with social media exposure which showed there are high prevalence of mental health problems, which positively associated with frequently SME during the COVID-19 outbreak .reasons explaining the association between frequently social media exposure and mental health. During the COVID-19 outbreak, disinformation and false reports about the COVID-19 have bombarded social media and stoked unfounded fears among many citizens ${ }^{(28)}$. 


\section{CONCLUSION}

HCWs showed mental disorders (fear, anxiety, and depression) scores more than non-HCW. Among HCW, females, the age group from 26 to 30 years, and more than $4 \mathrm{~h}$ of watching media regarding COVID-19 had significantly higher scores of fear, anxiety, and depression. Middle-educated participants had a significantly higher depression score. Non-married $\mathrm{HCW}$ showed a higher fear score. Among non-HCW, married and low educated levels expressed lower scores. Increasing hours of watching media was a significant predictor among $\mathrm{HCW}$ and non-HCW in predicting mental disorders scores.

Acknowledgment: We would like to thank all participants of this study.

Funding source: This research did not receive any specific grant from funding agencies in the public, commercial, or not-for-profit sectors.

Conflicts of interest: The authors declare that they have no conflict of interest.

Ethical approval: Approved.

\section{REFERENCES}

1. Nishiura $\mathbf{H}$, Jung $S$, Linton $\mathbf{N}$ et al. (2020): The extent of transmission of novel coronavirus in Wuhan, China, Journal of Clinical Medicine, 9(2): 330-33.

2. Chen N, Zhou M, Dong X et al. (2020): Epidemiological and clinical characteristics of 99 cases of 2019 novel coronavirus pneumonia in Wuhan, China: a descriptive study. The Lancet, 395(10223):507-13

3. Holshue M, DeBolt $\mathrm{C}$, Lindquist $\mathrm{S}$ et al. (2020): First case of 2019 novel coronavirus in the United States. New England Journal of Medicine, 382(10):929-936.

4. Wang C, Pan R, Wan X et al. (2020): Immediate psychological responses and associated factors during the initial stage of the 2019 coronavirus disease (COVID-19) epidemic among the general population in China. International Journal of Environmental Research and Public Health, 17(5):1729-34.

5. Fardin M (2020): COVID-19 and Anxiety: A Review of Psychological Impacts of Infectious Disease Outbreaks. Archives of Clinical Infectious Diseases, 15: 102779.

6. Makamure M, Mendiola W, Renteria D et al. (2013): A review of critical care nursing and disease outbreak preparedness. Dimensions of Critical Care Nursing, 32(4):157-61.

7. World Health Organization (2020): When and how to use masks. https://www.who.int/emergencies/diseases/novel-coronavirus2019/advice-for-public/when-and-how-to-use-masks.

8. Ho C, Chee C, Ho R (2020): Mental health strategies to combat the psychological impact of COVID-19 beyond paranoia and panic. Annals, Academy of Medicine Singapore, 49(3):155-160.

9. Rubin G, Wessely S (2020): The psychological effects of quarantining a city. doi: 10.1136/BMJ.m313. https://pubmed.ncbi.nlm.nih.gov/31992552/

10. Tucci V, Moukaddam N, Meadows J et al. (2017): The forgotten plague: Psychiatric manifestations of Ebola, Zika, and emerging infectious diseases. Journal of Global Infectious Diseases, 9(4):151-156.

11. Shigemura J, Ursano R, Morganstein $\mathbf{J}$ et al. (2020): Public responses to the novel 2019 coronavirus (2019-nCoV) in Japan: Mental health consequences and target populations. Psychiatry and Clinical Neurosciences, 74(4):281-282.

12. Lee S, Kang W, Cho A et al. (2018): Psychological impact of the 2015 MERS outbreak on hospital workers and quarantined hemodialysis patients. Comprehensive Psychiatry, 87:123-127.

13. Lai J, Ma S, Wang Y et al. (2020): Factors associated with mental health outcomes among health care workers exposed to Corona virus disease 2019. JAMA Network Open, 3(3): 203976.

14. Ornell F, Schuch J, Sordi A et al. (2020): Pandemic fear" and COVID-19: mental health burden and strategies. Brazilian Journal of Psychiatry, 42(3):232-235.

15. Ahorsu D, Lin C, Imani V et al. (2020): The fear of COVID-19 scale: development and initial validation. International Journal of Mental Health and Addiction, 20: 1-9.

16. Montazeri A, Vahdaninia M, Ebrahimi $M$ et al. (2003): The Hospital Anxiety and Depression Scale (HADS): translation and validation study of the Iranian version. Health and Quality of Life Outcomes, 1(1):14-18.

17. Lane F, To Y, Shelley K et al. (2012): An illustrative example of propensity score matching with education research. Career and Technical Education Research, 37(3):187-212.

18. Spoorthy M, Pratapa S, Mahant S (2020): Mental health problems faced by healthcare workers due to the COVID-19 pandemic-a review. Asian Journal of Psychiatry, 51(13):102119102123

19. Adams J, Walls $\mathbf{R}$ (2020): Supporting the health care workforce during the COVID-19 global epidemic. JAMA., 323(15):14391440 .

20. Kang L, Li Y, Hu S et al. (2020): The mental health of medical workers in Wuhan, China dealing with the 2019 novel coronavirus. The Lancet Psychiatry, 7(3):14-18.

21. Al-Rabiaah A, Temsah M, Al-Eyadhy A et al. (2020): Middle East Respiratory Syndrome-Corona Virus (MERS-CoV) associated stress among medical students at a university teaching hospital in Saudi Arabia. Journal of Infection and Public Health, 13(5):687-691.

22. Naushad V, Bierens J, Nishan K et al. (2019): A systematic review of the impact of the disaster on the mental health of medical responders. Prehospital and Disaster Medicine, 34(6):632-643.

23. Azimi A, Moayed M, Rahimibashar F et al. (2020): Compare the severity of psychological distress among four groups of Iranian society in the COVID-19 pandemic. BMC Psychiatry, 20: 402-405.

24. Liang Y, Chen M, Zheng $X$ et al. (2020): Screening for Chinese medical staff mental health by SDS and SAS during the outbreak of COVID-19. Journal of Psychosomatic Research, 133:110-113.

25. Peng E, Lee M, Tsai S et al. (2010): Population-based post-crisis psychological distress: an example from the SARS outbreak in Taiwan. Journal of the Formosan Medical Association, 109(7):524532.

26. Badahdah A, Khamis F, Al Mahyijari N (2020): The psychological well-being of physicians during COVID-19 outbreak in Oman. Psychiatry Research, 289:113053.

27. World Health Organization. (2020): Mental health and psychosocial considerations during the COVID-19 outbreak. https://www.unicef.org/ukraine/en/documents/mental-health-andpsychosocial-considerations-during-covid-19-outbreak

28. Gao J, Zheng P, Jia Y et al. (2020): Mental health problems and social media exposure during COVID-19 outbreak. Plos One Journal, 15(4):0231924. 